\title{
Kpc-scale radio-jets in narrow-line Seyfert 1 galaxies
}

\author{
Veeresh Singh* \\ Astronomy and Astrophysics Division, Physical Research Laboratory, Ahmedabad 380009, India \\ E-mail: veeresheprl.res.in
}

\section{Hum Chand}

Aryabhatta Research Institute of Observational Sciences (ARIES), Manora Peak, Nainital 263002 India

E-mail: humearies.res.in

\section{H. Ishwara-Chandra}

National Centre for Radio Astrophysics, TIFR, Post Bag 3, Ganeshkhind, Pune 411007, India

E-mail: ishwar@ncra.tifr.res.in

\section{Preeti Kharb}

National Centre for Radio Astrophysics, TIFR, Post Bag 3, Ganeshkhind, Pune 411007, India

E-mail: kharbancra.tifr.res.in

Narrow-Line Seyfert 1 galaxies (NLS1s) are generally believed to be radio-quiet Active Galactic Nuclei (AGN) with relatively less-massive $\left(10^{5}-10^{8} \mathrm{M}_{\odot}\right)$ Super-Massive Black Holes (SMBH). Using the FIRST radio detections of hitherto the largest sample of 11101 optically-selected NLS1s we find a rather uncommon population of 55 NLS1s with Kiloparsec-Scale Radio structures (KSRs). We note that the number of NLS1s with KSRs found in our study is only a lower limit considering the effects of sensitivity, spatial resolution and projection, and hence, the number of NLS1s with KSRs may be more common than thought earlier. The NLS1s with KSRs are distributed across a wide range of redshifts, flux densities and luminosities. NLS1s with KSRs tend to exhibit steep radio spectra and are likely to be misaligned version of blazar-like NLS1s. The ratio of IR to radio flux density $\left(\mathrm{q}_{22} \mu \mathrm{m}=\log \left[\mathrm{S}_{22} \mu \mathrm{m} / \mathrm{S}_{1.4 \mathrm{GHz}}\right]\right)$ versus radio luminosity plot suggests that KSRs are mostly powered by AGN, while KSRs in NLS1s with low radio luminosity $\left(\mathrm{L}_{1.4 \mathrm{GHz}}<10^{23.5} \mathrm{~W} \mathrm{~Hz}^{-1}\right)$ may have a contribution from circumnuclear starburst. The trend shown by KSRs in radio luminosity versus radio-size plot indicates that the radio-jets resulting in KSRs are either in the early phase of their evolution or inefficient to grow beyond the host galaxy.

Revisiting narrow-line Seyfert 1 galaxies and their place in the Universe - NLS1 Padova 9-13 April 2018

Padova Botanical Garden, Italy

\footnotetext{
* Speaker.
} 


\section{Introduction}

Seyfert 1 galaxies, a subclass of Active Galactic Nuclei (AGN), are generally characterized with the presence of broad emission lines (FWHM $\sim 5000 \mathrm{~km} \mathrm{~s}^{-1}$ ) in their optical spectra. However, a fraction of Seyfert 1 galaxies termed as Narrow-Line Seyfert 1 galaxies (NLS1s) exhibit relatively narrower (FWHM $<2000 \mathrm{~km} \mathrm{~s}^{-1}$ ) emission lines. Unlike Broad-Line AGN (BL-AGN), NLS1s show strong permitted Fe II emission lines, relatively weaker forbidden emission lines (e.g., [O III] $\lambda 5007 \AA / \mathrm{H} \beta<3$ ), steep soft X-ray spectra, and rapid X-ray variability [1]. Furthermore, it is believed that, compared to BL-AGN, NLS1s host less-massive $\left(10^{5}-10^{8} \mathrm{M}_{\odot}\right)$ SuperMassive Black Holes (SMBH) with relatively higher accretion rates [2]. Radio observations show that, in general, NLS1s are radio-quiet i.e., the ratio of radio flux density at $5 \mathrm{GHz}$ to the monochromatic optical flux at $4400 \AA\left(\mathrm{R}=\mathrm{S}_{5 \mathrm{GHz}} / \mathrm{S}_{4400}\right)$ is less than 10 [3]. However, in recent years the detections of several NLS1s in $\gamma$-ray with Fermi/LAT have shown that at least a small fraction of NLS1s possess strong radio-jets similar to blazars $[4,5]$. The existence of relativistic jets in many NLS1s is also confirmed by the direct imaging of parsec-scale radio jets using high-resolution Very Large Baseline Array (VLBA) observations [6]. We note that the high-resolution VLBA observations filter out the extended kiloparsec $(\mathrm{kpc})$-scale radio emission of low-surface-brightness. As per the orientation based unification model, the misaligned parent population of known blazar-like NLS1s with relativistic jets is expected to be several hundred. The relativistic radio-jets in misaligned sources are likely to give rise kpc-scale radio emission. However, in the literature, only a handful of NLS1s with KSRs are known [7, 8]. The scarcity of KSRs in NLS1s necessitates an investigation of kpc-scale radio emission in NLS1s using a statistically large sample. In this paper we attempt to investigate the properties of KSRs in NLS1s.

\section{Sample}

Our sample of 55 NLS1s with KSRs is extracted from [9]. Using 1.4 GHz Faint Images of the Radio Sky at Twenty-cm (FIRST), 1.4 GHz NRAO VLA Sky Survey (NVSS), 327 MHz Westerbork Northern Sky Survey (WENSS), and 150 MHz TIFR GMRT Sky Survey (TGSS), [9] found the radio detections of only 498/11101 ( 4.5\%) NLS1s in the sample of 11101 optically-selected NLS1s reported in [10]. The identification method of KSRs in FIRST radio sources includes (i) segregation of resolved and unresolved radio sources by accounting for the errors involved in fitting an elliptical Gaussian to the single component radio source, and then (ii) among the resolved sources a further criterion of $\mathrm{S}_{\text {int }} / \mathrm{S}_{\text {peak }}>1.12$ is applied to identify KSRs. A detailed description on the identification of KSRs and unresolved sources can be found in [9].

\section{Radio properties of NLS1s with KSRs}

Table 1 lists the range, median and standard deviation values of various distributions (i.e., projected linear radio size, redshift, $1.4 \mathrm{GHz}$ flux density, $1.4 \mathrm{GHz}$ radio luminosity, radio spectral index, radio-loudness parameter and $\mathrm{q}_{22} \mu \mathrm{m}$ ) for the NLS1s with KSRs and the NLS1s with no detected KSRs. 
Table 1: Radio properties of our sample of NLS1s.

\begin{tabular}{lccccr}
\hline Parameter & \multirow{2}{*}{ Unit } & \multicolumn{2}{c}{ NLS1s with KSRs } & \multicolumn{3}{c}{ NLS1s with no detected KSRs } \\
& & Range (median, std) & No. & Range (median, std) & No. \\
\hline Radio-size & $(\mathrm{kpc})$ & $1.13-1113.0(11.9,203)$ & 55 & $\ldots$ & 443 \\
Redshift & & $0.0098-0.7977(0.26,0.22)$ & 55 & $0.031-0.7969(0.36,0.22)$ & 443 \\
$\mathrm{~S}_{1.4 \mathrm{GHz}}$ & $(\mathrm{mJy})$ & $1.71-3733.7(5.56,496)$ & 55 & $1.0-8360.6(2.31,402.1)$ & 443 \\
$\operatorname{logL}_{1.4 \mathrm{GHz}}$ & $\left(\mathrm{W} \mathrm{Hz}^{-1}\right)$ & $21.85-26.48(24.05,1.34)$ & 55 & $21.89-27.14(24.0,1.01)$ & 443 \\
$\alpha_{150 \mathrm{GHz}}^{1.4 \mathrm{MHz}}$ & & $-1.19--0.31(-0.62,0.22)$ & 18 & $-1.13-0.57(-0.49,0.35)$ & 61 \\
$\operatorname{logR}{ }_{1.4 \mathrm{GHz}}$ & & $0.42-4.67(1.64,1.06)$ & 55 & $-0.02-5.39(1.46,0.88)$ & 443 \\
$\mathrm{q}_{22 \mu \mathrm{m}}$ & & $-2.39-1.61(1.04,0.90)$ & 38 & $-2.40-2.10(1.04,0.63)$ & 316 \\
\hline
\end{tabular}

Notes - std : standard deviation of the distribution. Spectral index between $150 \mathrm{MHz}$ and $1.4 \mathrm{GHz}$ is derived by assuming a power law $\left(\mathrm{S}_{v} \propto v^{\alpha}\right)$ radio spectrum.
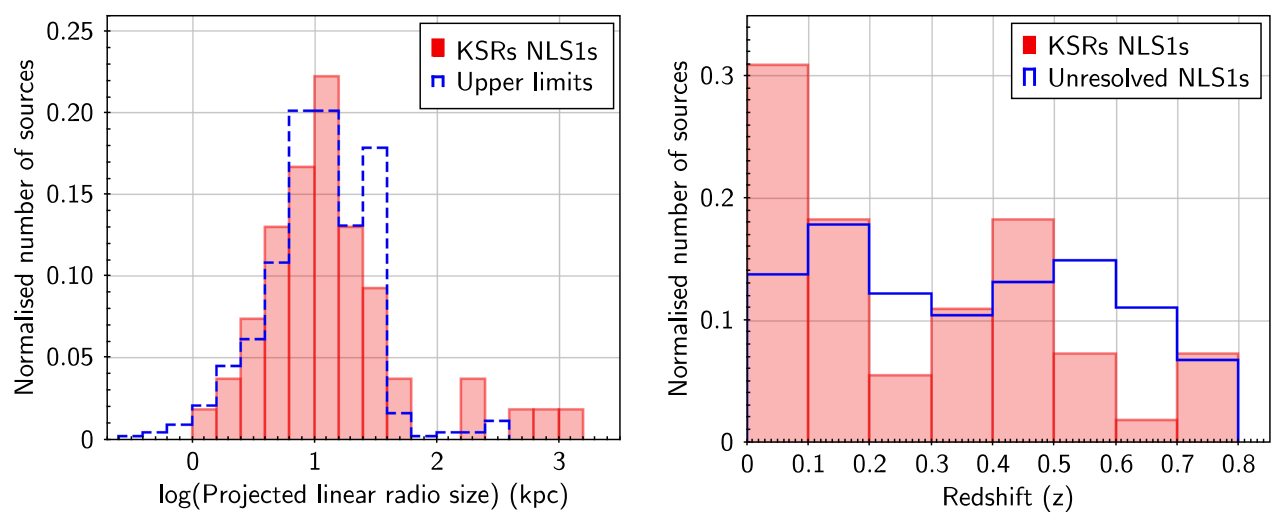

Figure 1: Left panel: The histogram of projected linear radio-size of KSRs and upper limits for unresolved sources. Right panel : Redshift distributions of KSRs and unresolved sources.
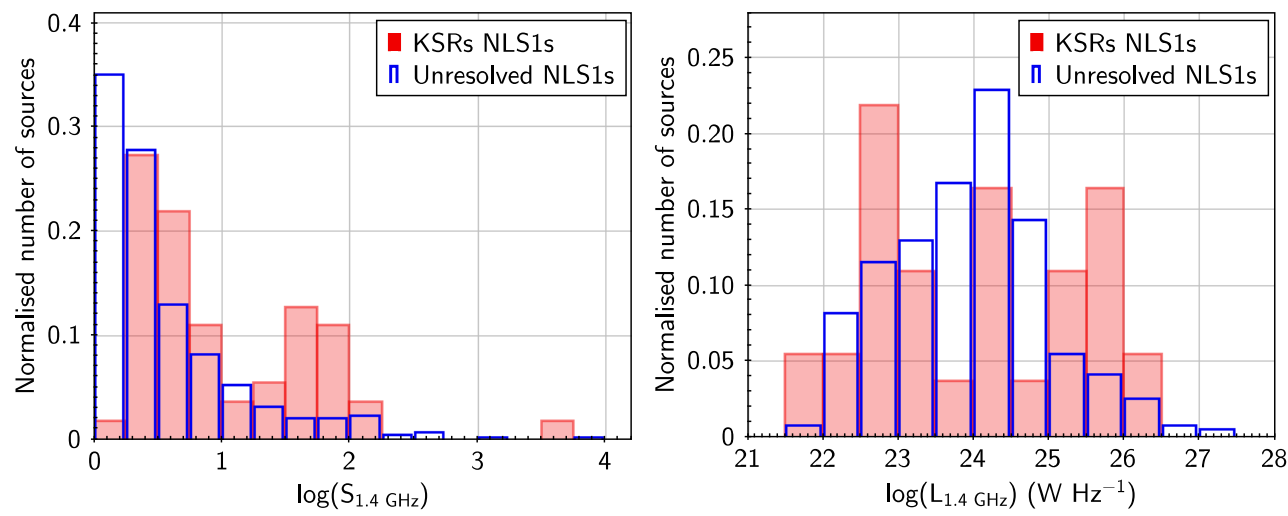

Figure 2: Left panel : $1.4 \mathrm{GHz}$ flux density distributions. Right panel : $1.4 \mathrm{GHz}$ radio luminosity distributions of KSRs and unresolved sources. 

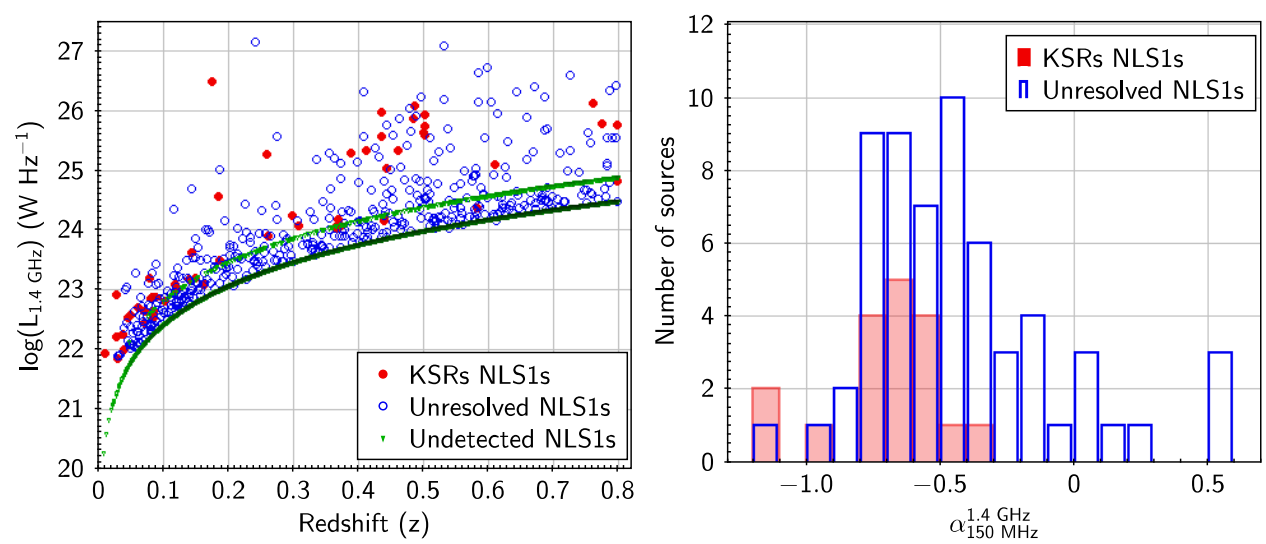

Figure 3: Left panel : Redshift versus $1.4 \mathrm{GHz}$ luminosity. The upper and lower green curves represent upper limits from NVSS and FIRST survey, respectively. Right panel: Two-point (150 MHz-1.4 GHz) spectral index distributions of KSRs and unresolved sources.
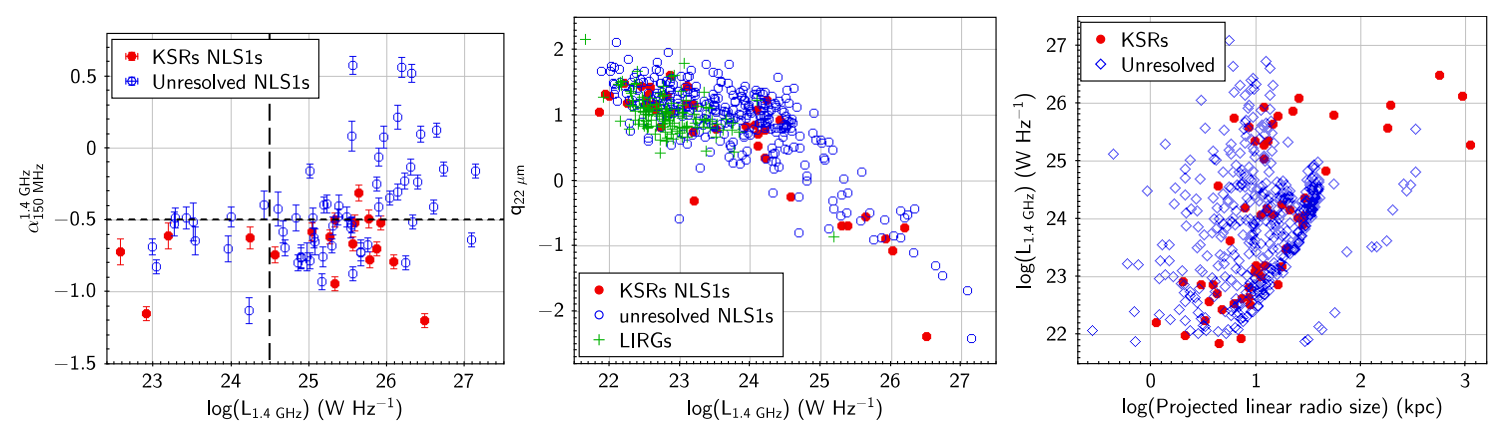

Figure 4: Left panel : $1.4 \mathrm{GHz}$ radio luminosity versus two-point spectral index. Middle panel : $\mathrm{q}_{22} \mu \mathrm{m}$ versus $1.4 \mathrm{GHz}$ radio luminosity. Right Panel : Projected linear radio-size versus $1.4 \mathrm{GHz}$ radio luminosity plot.

\subsection{Kiloparsec-Scale Radio structures (KSRs) in NLS1s}

We estimate the radio-sizes of 55 NLS1s with resolved radio emission and upper limits for 443 NLS1s with unresolved radio emission using FIRST observations whenever available, otherwise NVSS measurements are used. We find that 55 NLS1s with resolved radio emission have radiosize ranging from $1.1 \mathrm{kpc}$ to $1.1 \mathrm{Mpc}$ with a median of $11.3 \mathrm{kpc}$, hence confirming that a small fraction $(55 / 11101 \sim 0.5 \%)$ of NLS1s possess KSRs (see Fig. 1, left panel and Table 1). We note that the number of NLS1s with KSRs found in our study is only a lower limit as sources appearing unresolved, particularly at higher redshifts, may possess KSRs and jet-like KSRs aligned close to the line-of-sight would appear foreshortened due to projection effect. Notably, our study reveals the highest number of NLS1s with KSRs till date. It is worth noting that barring a few NLS1s with radio-size $\geq 100 \mathrm{kpc}$, most of the NLS1s have radio-sizes (ranging from $1.1 \mathrm{kpc}$ to $30 \mathrm{kpc}$ ) smaller than the average optical size of the host galaxy. Thus, the radio-size of KSRs in NLS1s seem similar to those found in Seyfert galaxies [11]. Previous studies have shown that the radio emission in NLS1s is mostly parsec-scale compact [6] and KSRs are rare [7]. Although, recently using deep $(5 \sigma \sim 50 \mu \mathrm{Jy})$ and high-resolution $\left(0^{\prime \prime} .5\right) 5 \mathrm{GHz}$ VLA A-configuration radio observations [8] have 
found KSRs in 21 sources among the sample of 74 NLS1s. Therefore, deep arcsec-resolution radio observations of our NLS1s can detect more KSRs.

\subsection{Redshift and $1.4 \mathrm{GHz}$ radio flux densities}

The redshift distribution 55 NLS1s with KSRs spans over 0.0098 to 0.7977 with a median of 0.26 , while 443 NLS1s with unresolved radio counterparts are distributed over the same range but with a higher median of 0.36 (see Fig. 1, right panel). It is evident that KSRs are preferentially found in NLS1s at lower redshifts, which can be due to an observational bias i.e., the radio structures of similar scale tend to have smaller apparent angular sizes with the increase in redshift. We note that the KSRs are found in both faint as well as bright radio sources. The $1.4 \mathrm{GHz}$ flux densities of NLS1s with KSRs range from $1.71 \mathrm{mJy}$ to $3733.7 \mathrm{mJy}$ with a median of $5.56 \mathrm{mJy}$, while $1.4 \mathrm{GHz}$ flux densities of NLS1s with unresolved emission span over a much larger range of 1.0 mJy to 8360.6 mJy but with a lower median of $2.31 \mathrm{mJy}$ (see Fig. 2, left panel). Thus, most of the sources at the very faint end $\left(\mathrm{S}_{1.4 \mathrm{GHz}} \leq 2.5 \mathrm{mJy}\right)$ appear unresolved.

\subsection{Radio luminosities and spectra}

1.4 GHz radio luminosity distribution for both unresolved and KSRs NLS1s span over a wide range $\left(10^{21}-10^{27} \mathrm{~W} \mathrm{~Hz}^{-1}\right)$ with a median of $\sim 10^{24} \mathrm{~W} \mathrm{~Hz}^{-1}$ (see Fig. 2, right panel). Moreover, unlike unresolved radio sources, the radio luminosity distribution of KSRs sources shows three apparent peaks at low $\left(\mathrm{L}_{1.4 \mathrm{GHz}}=10^{22.75} \mathrm{~W} \mathrm{~Hz}^{-1}\right)$, moderate $\left(\mathrm{L}_{1.4 \mathrm{GHz}}=10^{24.25} \mathrm{~W} \mathrm{~Hz}^{-1}\right)$ and high $\left(\left(\mathrm{L}_{1.4 \mathrm{GHz}}=10^{25.75} \mathrm{~W} \mathrm{~Hz}^{-1}\right)\right.$ luminosity. We note that, on average, KSRs sources with low, moderate and high radio luminosities are found at low $\left(z_{\text {median }} \sim 0.1\right)$, moderate $\left(z_{\text {median }} \sim 0.3\right)$ and high redshift $\left(z_{\text {median }} \sim 0.5\right.$ ), respectively (see Fig. 3, left panel). The prevalence of radio powerful sources at higher redshifts is consistent with the fact that co-moving density of radio-powerful AGN increases with redshift over $z \sim 0.0-2.0$. However, three peaks in the radio luminosity distribution of KSR sources appear intriguing and deep radio observations of a large sample of NLS1s are required to confirm the existence of these peaks. Further, we note that the KSRs are present in both radio-quiet and radio-loud NLS1s. Although, the median value of radio-loudness for NLS1s with KSRs is marginally higher than that for the NLS1s with unresolved radio emission (see Table 1). We estimate two-point spectral index $\left(\alpha_{150 \mathrm{MHz}}^{1.4 \mathrm{GHz}}\right)$ for KSRs and unresolved sources using $1.4 \mathrm{GHz}$ flux density from FIRST or NVSS and $150 \mathrm{MHz}$ flux density from TGSS. The distribution of $\alpha_{150 \mathrm{MHz}}^{1.4 \mathrm{GHz}}$ shows that spectral indices for $18 \mathrm{KSR}$ sources range from -1.19 to -0.31 with a median of -0.61 , while 61 unresolved radio sources have spectral indices spanning over -1.13 to 0.57 with a median of -0.49 (see Fig. 3, right panel). The steeper radio spectra of KSRs are consistent with the optically-thin synchrotron radio emission, while compact sources can have flat/inverted radio spectrum due to free-free or synchrotron self-absorption. The very steep $\left(\alpha_{150}^{1.4 \mathrm{GHz}}<-1.0\right)$ radio spectra can also be indicative of the relic radio emission caused by the previous episode of AGN activity $[12,13]$. From luminosity versus spectral index plot (Fig. 4, left panel) it is apparent that the KSRs sources distributed across all luminosities have steep spectra, while unresolved sources show a clear trend of flattening of radio spectra at higher luminosities. The unresolved sources with high radio luminosity and flat/inverted radio spectra can be blazar-like sources, in which luminosity is boosted due to relativistic beaming effect. 


\subsection{Origin and evolution of KSRs in NLS1s}

We attempt to investigate whether KSRs are powered by AGN or star-formation by using the radio-IR correlation which can also be represented as the ratio of IR to radio flux density i.e., $\mathrm{q}_{22} \mu \mathrm{m}$ $=\log \left[\mathrm{S}_{22} \mu \mathrm{m} / \mathrm{S}_{1.4 \mathrm{GHz}}\right]$, where IR flux at $22 \mu \mathrm{m}$ is taken from Wide-field Infrared Survey Explorer (WISE). Star-forming galaxies are known to show a tight correlation between IR and radio emission while radio-loud AGN tend to deviate. In Fig. 4 (right panel) we plot $\mathrm{q}_{22} \mu \mathrm{m}$ versus $1.4 \mathrm{GHz}$ radio luminosity for NLS1s with KSRs and with unresolved radio emission and Luminous InfraRed Galaxies (LIRGs). It is evident that $\mathrm{q}_{22} \mu \mathrm{m}$ decreases with the increase in radio luminosity. All the radio-luminous NLS1s $\left(\mathrm{L}_{1.4 \mathrm{GHz}}>10^{23.5} \mathrm{~W} \mathrm{~Hz}^{-1}\right.$ ) have $\mathrm{q}_{22} \mu \mathrm{m}$ much lower than that for star-forming LIRGs. Therefore, KSRs in radio-luminous NLS1s is likely to be driven by AGN. However, a fraction of NLS1s with lower radio luminosities show $\mathrm{q}_{22} \mu \mathrm{m}$ similar to LIRGs, and hence, KSRs in these NLS1s can have a contribution from star-formation. Although, the small radio sizes of NLS1s with low radio luminosity (Fig. 4, right panel) indicate that the radio emission is driven by either AGN or a compact circumnuclear starburst.

From Fig. 4 (right panel) it is apparent that radio-size increases with the increase in radio luminosity except for very large evolved radio sources $(>100 \mathrm{kpc})$. The trend shown by KSRs in radio luminosity versus projected linear radio-size is consistent with the dynamical evolutionary models, suggesting that the compact parsec-scale radio-jets evolve into kpc-scale radio-jets which eventually turn into large radio galaxies displaying hundreds of kpc jet-lobe structures [14]. Therefore, the radio jets of a few kpc scale in NLS1s may be in the early phase of evolution similar to Giga-hertz Peaked Spectrum (GPS) and Compact Steep Spectrum (CSS) radio sources [15, 16]. However, the lack of large KSRs (>100) in NLS1s infers that radio-jets in many NLS1s may not be sufficiently powerful to dispel the Inter-Stellar Medium (ISM), and hence, may remain confined within the host galaxy.

\section{Conclusions}

Using FIRST radio detections of hitherto the largest optically-selected sample of 11101 NLS1s we obtain the largest sample of 55 NLS1s with KSRs. The NLS1s with KSRs are distributed across a wide range of redshifts, flux densities and luminosities. Unlike unresolved radio sources, the KSRs tend to show steep radio spectra, therefore NLS1s with KSRs are likely to be misaligned version of the flat-spectrum radio-loud NLS1s. The KSRs are mostly powered by AGN while they can have a contribution from circumnuclear starburst in NLS1s with low radio luminosities. The radio luminosity versus radio size plot suggests that the radio-jet driving KSRs in NLS1s are either in the early phase of their evolution or are frustrated by the ISM.

\section{Acknowledgements}

VS acknowledges support from PRL Ahmedabad, and the RadioNet. This conference has been organized with the support of the Department of Physics and Astronomy "Galileo Galilei", the University of Padova, the National Institute of Astrophysics INAF, the Padova Planetarium, and the RadioNet consortium. RadioNet has received funding from the European Union's Horizon 2020 research and innovation programme under grant agreement No 730562. 


\section{References}

[1] F. Panessa, A. de Rosa, L. Bassani, A. Bazzano, A. Bird, R. Landi et al., Narrow-line Seyfert 1 galaxies at hard X-rays, MNRAS 417 (2011) 2426 [1106.3023].

[2] L. Foschini, M. Berton, A. Caccianiga, S. Ciroi, V. Cracco, B. M. Peterson et al., Properties of flat-spectrum radio-loud narrow-line Seyfert 1 galaxies, A\&A 575 (2015) A13 [1409.3716].

[3] S. Komossa, W. Voges, D. Xu, S. Mathur, H.-M. Adorf, G. Lemson et al., Radio-loud Narrow-Line Type 1 Quasars, aj 132 (2006) 531 [astro-ph/ 0603680 ].

[4] A. A. Abdo, M. Ackermann, M. Ajello, L. Baldini, J. Ballet, G. Barbiellini et al., Radio-Loud Narrow-Line Seyfert 1 as a New Class of Gamma-Ray Active Galactic Nuclei, ApJ 707 (2009) L142 [0911.3485].

[5] F. D’Ammando, M. Orienti, J. Finke, C. M. Raiteri, T. Hovatta, J. Larsson et al., The most powerful flaring activity from the NLSy1 PMN J0948+0022, MNRAS 446 (2015) 2456 [1 410 . 7144 ].

[6] M. Gu, Y. Chen, S. Komossa, W. Yuan, Z. Shen, K. Wajima et al., The Radio Properties of Radio-loud Narrow-line Seyfert 1 Galaxies on Parsec Scales, ApJS 221 (2015) 3 [1509. 01889].

[7] J. L. Richards and M. L. Lister, Kiloparsec-Scale Jets in Three Radio-Loud Narrow-Line Seyfert 1 Galaxies, ApJ 800 (2015) L8 [1501.05299].

[8] M. Berton, E. Congiu, E. Järvelä, R. Antonucci, P. Kharb, M. L. Lister et al., Radio-emitting narrow-line Seyfert 1 galaxies in the JVLA perspective, A\&A 614 (2018) A87 [1801.03519].

[9] V. Singh and H. Chand, Investigating kpc-scale radio emission properties of narrow-line Seyfert 1 galaxies, ArXiv e-prints (2018) [1807.01945].

[10] S. Rakshit, C. S. Stalin, H. Chand and X.-G. Zhang, A Catalog of Narrow Line Seyfert 1 Galaxies from the Sloan Digital Sky Survey Data Release 12, ApJS 229 (2017) 39 [170 407700 ].

[11] V. Singh, C. H. Ishwara-Chandra, Y. Wadadekar, A. Beelen and P. Kharb, Kiloparsec-scale radio emission in Seyfert and LINER galaxies, MNRAS 446 (2015) 599 [1410 . 2720].

[12] P. Kharb, S. Srivastava, V. Singh, J. F. Gallimore, C. H. Ishwara-Chandra and H. Ananda, A GMRT study of Seyfert galaxies NGC 4235 and NGC 4594: evidence of episodic activity?, MNRAS 459 (2016) 1310 [1603.08364].

[13] E. Congiu, M. Berton, M. Giroletti, R. Antonucci, A. Caccianiga, P. Kharb et al., Kiloparsec-scale emission in the narrow-line Seyfert 1 galaxy Mrk 783, A\&A 603 (2017) A32 [1 704.03881 ].

[14] I. A. G. Snellen, R. T. Schilizzi, G. K. Miley, A. G. de Bruyn, M. N. Bremer and H. J. A. Röttgering, On the evolution of young radio-loud AGN, MNRAS 319 (2000) 445 [astro-ph/ 0002130 ].

[15] A. Caccianiga, D. Dallacasa, S. Antón, L. Ballo, M. Berton, K.-H. Mack et al., SDSSJ143244.91+301435.3 at VLBI: a compact radio galaxy in a narrow-line Seyfert 1, MNRAS 464 (2017) 1474 [1609.09501].

[16] M. Berton, A. Caccianiga, L. Foschini, B. M. Peterson, S. Mathur, G. Terreran et al., Compact steep-spectrum sources as the parent population of flat-spectrum radio-loud narrow-line Seyfert 1 galaxies, A\&A 591 (2016) A98 [1601.06165]. 